\title{
Regularity of the Scattering Trajectories in Classical Mechanics
}

\section{Eugene Gutkin*}

Department of Mathematics, University of Southern California, Los Angeles, CA 90089-1113, USA

\begin{abstract}
We establish conditions under which all trajectories of a mechanical system have a regular scattering behavior. Applications to the many-body problems on the line and to the systems with exponential cone potentials are worked out.
\end{abstract}

\section{Introduction}

To motivate our study let us consider the classical motion of $n d$-dimensional particles with pairwise interactions. We consider the situation where the forces of interaction are central, conservative and repulsive. Denote by $x_{1}, \ldots, x_{n}$ the positions and by $\dot{x}_{1}, \ldots, \dot{x}_{n}$ the velocities of the particles. Let $m_{1}, \ldots, m_{n}$ be their masses and $v_{i j} \geqq 0,1 \leqq i<j \leqq n$, the potentials of interaction. Then the total energy is

$$
E(x, \dot{x})=\frac{1}{2} \sum_{i=1}^{n} m_{i}\left\|\dot{x}_{i}\right\|^{2}+\sum_{i<j} v_{i j}\left(\left\|x_{i}-x_{j}\right\|\right) .
$$

It is known $[1,9]$ that any motion $\left\{x_{1}(t), \ldots, x_{n}(t) ;-\infty<t<\infty\right\}$ has asymptotic velocities at infinity

$$
\dot{x}_{i}(\infty)=\lim _{t \rightarrow \infty} \dot{x}_{i}(t), \quad i=1, \ldots, n .
$$

Assume that the pair potentials satisfy the fast decay condition

$$
\int_{\rho}^{\infty} v_{i j}(r) d r<\infty
$$

It was shown in [2] that the motions with distinct asymptotic velocities

$$
\dot{x}_{1}(\infty) \neq \cdots \neq \dot{x}_{n}(\infty)
$$

* Partially supported by NSF Grant DMS 8600350 
have the asymptotics

$$
x_{i}(t)=\dot{x}_{i}(\infty) t+a_{i}+o(1), \quad i=1, \ldots, n .
$$

Such motions are called asymptotically uniform.

Presumably, (1.4) is satisfied for a dense open set $D_{+} \subset R^{2 d n}$ of initial data which defines the mapping

$$
W_{+}: D_{+} \rightarrow R^{2 d n}
$$

assigning, by (1.5), to a point $(x(0), \dot{x}(0))$ in $D_{+}$the asymptotic data $\left.(\dot{x}(\infty)), a\right)$ of the corresponding trajectory. The operator $W_{+}$and its counterpart at minus infinity

$$
W_{-}: D_{-} \rightarrow R^{2 d n}
$$

allow us to define the scattering $S=W_{+} W_{-}^{-1}$.

The study of the $W_{ \pm}$and $S$ is inconvenienced by the fact that, in general, $D_{+} \neq D_{-} \neq R^{2 d n}$, which is related to the phenomenon that not all trajectories satisfy (1.4). It is natural to call motions satisfying (1.4) and (1.5) regular.

However, it was discovered in the course of investigation of integrable manybody problems that some of them have only regular trajectories. For instance, such is the Calogero-Moser system of $n$ particles on the line interacting via the $x^{-2}$ potential and the Toda lattice [8].

In this paper we, developing an idea of [8], establish sufficient conditions for a scattering problem to have only regular trajectories. Applying these conditions we show that for many natural classes of scattering problems all motions are regular. This is true, in particular, for exponential potentials (Corollary 3) and for one-dimensional many-body problems (Theorems 5 and 6). A different approach to the regularity of trajectories for the many-body scattering in one dimension is developed by A. Hubacher [7], see remark at the end of Sect. 3.

\section{Conditions for Regularity}

We consider Newton's equation of motion

$$
M \ddot{x}=F(x), \cdot
$$

where $x=x(t) \in R^{N}, M>0$ is the mass matrix and

$$
F(x)=-\operatorname{grad} V(x)
$$

is the conservative force corresponding to the potential $V$. The present work relies to some extent on [3-6] where only the case $M=1$ was considered. It will be shown in this section how to remove this restriction, so the results of [3-6] remain valid for any positive mass matrix $M$.

We need to establish some terminology and notation. The cone spanned by vectors $e_{i} \in R^{N}, i \in I$, is the closure of the set $\left\{x=\sum c_{i} e_{i}, i \in I, c_{i} \geqq 0\right\}$ as opposed to the subspace spanned by $e_{i}, i \in I$. A cone $C$ in $R^{N}$ is called proper if it does not contain a straight line. Let $\langle x, y\rangle$ be the scalar product in $R^{N}$. The dual cone $C^{*}$ of $C$ is defined by $C^{*}=\{y:\langle y, x\rangle \geqq 0$ for all $x \in C\}$. A closed convex cone is proper if and only if $C^{*}$ spans $R^{N}$. By Int $X$ we denote the interior of $X$. 
We consider potentials $V$ that satisfy the following conditions.

C.1. $V \geqq 0$ is defined and continuously differentiable in a convex open domain $\Omega$. The vectors - grad $V(x), x \in \Omega$, span a proper cone $C$. Domain $\Omega$ is closed under $x \rightarrow x+C^{*}$.

C.2. For any $0 \neq e \in C$ there is $l \geqq-\infty$ such that for $x \in \Omega,\langle x, e\rangle>l$ and $\langle x, e\rangle \rightarrow l$ implies $V(x) \rightarrow \infty$. The function $\|\operatorname{grad} V(x)\|$ is bounded away from zero in any domain $\{x \in \Omega:\langle x, e\rangle\langle L, 0 \neq e \in C\}$.

C.3. Let $e^{*} \in C^{*}$ and let $C_{0}=\left\{e \in C:\left\langle e, e^{*}\right\rangle=0\right\}$. For any $e^{*} \in C^{*}$ there is a closed convex cone $C_{1} \subset C$ such that $e^{*} \in \operatorname{Int} C_{1}^{*}$, and the decomposition

$$
V=V_{0}+V_{1}
$$

where $-\operatorname{grad} V_{0}(x) \in C_{0},-\operatorname{grad} V_{1}(x) \in C_{1}$ and

$$
\int_{0}^{\infty} V_{1}\left(a+r e^{*}\right) d r<\infty
$$

for any $a \in \Omega$.

Potentials satisfying C.1 were dubbed in [4] the cone potentials.

Theorem 1. Let potential $V$ satisfy C.1, C.2 and C.3. Then

i) for any trajectory $x(t)-\infty<t<\infty$, of (2.1) the asymptotic velocity $\dot{x}(\infty)=$ $\lim \dot{x}(t)$ exists and $\dot{x}(\infty) \in \operatorname{Int} C^{*}$.

$t \rightarrow \infty$.i) Any trajectory is asymptoticc

ii) Any trajectory is asymptotically uniform, i.e. it has the asymptotics as $t \rightarrow \infty$,

$$
x(t)=\dot{x}(\infty) t+a+o(1) .
$$

iii) The asymptotic velocity $\dot{x}(-\infty)$ exists for any trajectory and $\dot{x}(-\infty)$ $\epsilon-$ Int $C$. Every motion is asymptotically uniform at $t \rightarrow-\infty$.

Proof. Assume first that $M=1$. By [3], for any trajectory $x(t)$ the limit $\dot{x}(\infty)$ exists and $\dot{x}(\infty) \in C^{*}$. If $\dot{x}(\infty) \in \operatorname{Int} C^{*}$ then, by Theorem 1 of [4], (2.5) holds. Hence, it remains to show that $\dot{x}(\infty) \in \operatorname{Int} C^{*}$ for any trajectory.

Assume the opposite and denote $\dot{x}(\infty)$ by $e^{*}$. Consider the cones $C_{0}$ and $C_{1}$ and the decomposition (2.3). Set $F_{0}=-\operatorname{grad} V_{0}, F_{1}=-\operatorname{grad} V_{1}$. Rewrite (2.1) as

$$
\ddot{x}(t)=F_{0}(x(t))+F_{1}(x(t)) .
$$

Since $e^{*} \in \operatorname{Int} C_{1}^{*}$, there is a positive constant a such that for any $e_{1} \in C_{1}$,

$$
\left\|e_{1}\right\| \leqq a\left\langle e_{1}, e^{*}\right\rangle \text {. }
$$

Note that inequality (2.7) is equivalent to $e^{*} \in \operatorname{Int} C_{1}^{*}$.

Since $\dot{x}(t) \rightarrow e^{*}$ for any $\varepsilon>0$ there exists $t_{0}$ such that $\left\|\dot{x}(t)-e^{*}\right\|<\varepsilon$ for $t>t_{0}$. For any vector $e_{1} \in C_{1}$ and $t>t_{0}$,

$$
\begin{aligned}
\left\langle e_{1},\left(\dot{x}(t)-e^{*} / 2\right)\right\rangle & =\frac{1}{2}\left\langle e_{1}, e^{*}\right\rangle+\left\langle e_{1}, \dot{x}(t)-e^{*}\right\rangle \geqq \frac{1}{2}\left\langle e_{1}, e^{*}\right\rangle-\left|\left\langle e_{1}, \dot{x}(t)-e^{*}\right\rangle\right| \\
& \geqq \frac{1}{2 a}\left\|e_{1}\right\|-\varepsilon\left\|e_{1}\right\|=\left(\frac{1}{2 a}-\varepsilon\right)\left\|e_{1}\right\| .
\end{aligned}
$$


Choose $\varepsilon<1 / 2 a$. By (2.8), for $t>t_{0}, \dot{x}(t)-\frac{1}{2} e^{*} \in \operatorname{Int} C_{1}^{*}$ and

$$
\left\langle e_{1}, \dot{x}(t)\right\rangle>\frac{1}{2}\left\langle e_{1}, e^{*}\right\rangle \geqq \frac{1}{2 a}\left\|e_{1}\right\| .
$$

Applying inequality (2.9) to $e_{1}=F_{1}(x(\tau))$, we have, for $t_{0}<t<\infty$,

$$
\begin{aligned}
\left\|\int_{t}^{\infty} F_{1}(x(\tau)) d \tau\right\| & \leqq \int_{t}^{\infty}\left\|F_{1}(x(\tau))\right\| d \tau \leqq 2 a \int_{t}^{\infty}\left\langle F_{1}(x(\tau)), \dot{x}(\tau)\right\rangle d \tau \\
& =-2 a \int_{t}^{\infty} \frac{d V_{1}(x(\tau))}{d \tau}-d \tau=2 a V_{1}(x(t)) .
\end{aligned}
$$

Thus the integral

$$
G_{1}(t)=\int_{t}^{\infty} F_{1}(x(\tau)) d \tau
$$

converges absolutely and, for $t>t_{0}$

$$
\left\|G_{1}(t)\right\| \leqq \text { const } V_{1}(x(t)) .
$$

Since $F_{1}(x) \in C_{1}$, for any two vectors $a, b$ such that $b-a \in C_{1}^{*}$, we have

$$
V_{1}(a)-V_{1}(b)=\int_{0}^{1}\left\langle F_{1}(a+s(b-a)), b-a\right\rangle d s \geqq 0 .
$$

Set $a=\frac{1}{2} e^{*}\left(t-t_{0}\right)+x\left(t_{0}\right), b=x(t)$. Then

$$
b-a=\int_{t_{0}}^{t} \frac{d}{d \tau}\left[x(\tau)-\frac{1}{2} e^{*}\left(\tau-t_{0}\right)-x\left(t_{0}\right)\right] d \tau=\int_{t_{0}}^{t}\left[\dot{x}(\tau)-\frac{1}{2} e^{*}\right] d \tau .
$$

Since, for $\tau>t_{0}, \dot{x}(\tau)-\frac{1}{2} e^{*}$ belongs to $C_{1}^{*}$, by (2.13), for $t_{0}<t<\infty$,

$$
x(t)-\frac{1}{2} e^{*}\left(t-t_{0}\right)-x\left(t_{0}\right) \in C_{1}^{*}
$$

and, in view of (2.12),

$$
V_{1}(x(t)) \leqq V_{1}\left(\frac{1}{2} e^{*}\left(t-t_{0}\right)+x\left(t_{0}\right)\right) .
$$

Inequalities (2.11) and (2.14) yield

$$
\left\|G_{1}(t)\right\| \leqq \text { const } V_{1}\left(x\left(t_{0}\right)+\frac{1}{2} e^{*}\left(t-t_{0}\right)\right)
$$

We have from (2.6) and (2.10),

$$
\frac{d}{d t}\left(\dot{x}(t)+G_{1}(t)\right)=F_{0}(x(t))
$$

Since $C_{0}$ is a closed convex nonempty proper cone, the intersection Int $C_{0} \cap \operatorname{Int} C_{0}^{*}$ is not empty. Choose a vector $e$ from it and set

$$
g(t)=\left\langle\dot{x}(t)+G_{1}(t), e\right\rangle .
$$

By (2.16),

$$
\dot{g}(t)=\left\langle F_{0}(x(t)), e\right\rangle \geqq 0,
$$


hence $g$ is monotonically increasing. By (2.4) and (2.15), $G_{1}(t)$ goes to zero as $t \rightarrow \infty$, hence

$$
\lim _{t \rightarrow \infty} g(t)=\left\langle e^{*}, e\right\rangle=0
$$

By (2.18) and (2.19),

$$
g(t) \leqq 0
$$

By (2.15) and (2.4), the integral

$$
H_{1}(t)=\int_{t}^{\infty} G_{1}(\tau) d \tau
$$

converges, and $H_{1}(t) \rightarrow 0$ as $t \rightarrow \infty$. Set

$$
h(t)=\left\langle x(t)-H_{1}(t), e\right\rangle .
$$

Then $\dot{h}(t)=g(t)$ and, in view of (2.20), $h$ is monotonically decreasing. Hence, $h(t)$ is bounded above as $t \rightarrow \infty$. Since $H_{1}(t) \rightarrow 0,\langle x(t), e\rangle$ is bounded above as $t \rightarrow \infty$. By C.2, $\|F(x(t))\|$ is bounded away from zero. Since $F_{1}(x(t)) \rightarrow 0,\left\|F_{0}(x(t))\right\|$ is bounded away from zero as $t \rightarrow \infty$. Since $F_{0} \in C_{0}$ and $e \in \operatorname{Int} C_{0}^{*}$, by (2.7), there is a positive constant such that

$$
\left\langle F_{0}(x(t)), e\right\rangle \geqq \mathrm{const}\left\|F_{0}(x(t))\right\|
$$

and $\left\langle F_{0}(x(t)), e\right\rangle$ is bounded away zero as $t \rightarrow \infty$. This implies, in view of (2.18), that $g(t) \rightarrow \infty$ which contradicts $(2.20)$ and proves the first two assertions of the theorem. The third one can be proved by the same argument considering $t \rightarrow-\infty$. It also follows from i) and ii) by the change of variables $(x, t) \rightarrow(-x,-t)$.

Let now $M>0$ be arbitrary. Set

$$
y=M^{1 / 2} x, \quad \tilde{V}(y)=V\left(M^{-1 / 2} y\right) .
$$

Then (2.1) is equivalent to

with

$$
\ddot{y}=\tilde{F}(y)
$$

$$
\tilde{F}(y)=-\operatorname{grad} \tilde{V}(y)=M^{-1 / 2} F\left(M^{-1 / 2} y\right) .
$$

Set $\tilde{C}=M^{-1 / 2} C, \widetilde{\Omega}=M^{1 / 2} \Omega$. The cone $\tilde{C}$ is proper and $\tilde{C}^{*}=M^{1 / 2} C^{*}$. It is straightforward but tedious (we leave it to the reader) to check that the potential $\widetilde{V}$, the cone $\widetilde{C}$ and the domain $\widetilde{\Omega}$ satisfy conditions C.1-C.3.

In view of the above, Theorem 1 holds for the trajectories $y(t)$ of (2.25). Since the trajectories $x(t)$ of $(2.1)$ and $y(t)$ are related by

$$
x(t)=M^{1 / 2} y(t)
$$

Theorem 1 follows.

Now we give the formal definition of regular motions in classical scattering.

Definition 1. A trajectory $x(t),-\infty<t<\infty$, of $(2.1)$ is called regular if it has the asymptotics (as $t \rightarrow \pm \infty$ )

$$
x(t)=b_{ \pm} t+a_{ \pm}+o(1), \quad \dot{x}(t)=b_{ \pm}+o(1)
$$


with $b_{+}, b_{-} \neq 0$. The vectors $b_{+}, b_{-}$and $a_{+}, a_{-}$are the asymptotic velocities and phases at $t=+\infty$ and $t=-\infty$ respectively.

Theorem 1 contains the following.

Corollary 1. If potential $V$ satisfies conditions C.1-C.3 then all motions of the dynamics (2.1) are regular.

The following corollary has been proved in the course of proof of Theorem 1.

Corollary 2. Assume that conditions C.1, C.2 and C.3 are satisfied. Then any trajectory $x(t)$ has asymptotics $(2.28)$ with $b_{ \pm} \in \pm$ Int $C^{*}$. There exists $t_{0}>0$ such that for $t>t_{0}$

$$
V(x(t)) \leqq \text { const } V\left(x\left(t_{0}\right)+\frac{1}{2} b_{+}\left(t-t_{0}\right)\right)
$$

and for $t<-t_{0}$

$$
V(x(t)) \leqq \text { const } V\left(x\left(t_{0}\right)+\frac{1}{2} b_{-}\left(t+t_{0}\right)\right) .
$$

For some applications, condition C.3 is too restrictive. It can be replaced by the following two conditions.

C.4. For any $e \in \operatorname{Int} C^{*}$ and $a \in \Omega$,

$$
\int_{0}^{\infty} V\left(a+r e^{*}\right) d r<\infty
$$

C.5. Let $x(t)$ be a trajectory and let $e^{*} \in C^{*}$ be its asymptotic velocity (at plus infinity). Let $C_{0} \subset C$ be the subcone of vectors orthogonal to $e^{*}$. Then there is a decomposition

$$
F(x)=F_{0}+F_{1}(x)
$$

where $F_{0}(x) \in C_{0}$ and

$$
\int_{-\infty}^{\infty}|t|\left\|F_{1}(x(t))\right\| d t<\infty .
$$

Proposition 1. Let a potential $V$ satisfy conditions C.1, C.2, C.4 and C.5. Then every motion of the dynamics $(2.1)$ is regular, with $\dot{x}(\infty) \in \operatorname{Int} C^{*}, \dot{x}(-\infty) \in-\operatorname{Int} C^{*}$.

Proof. Following the proof of Theorem 1 we come to the point where we need to show that $\dot{x}(\infty) \in \operatorname{Int} C^{*}$. Assume the opposite and set $\dot{x}(\infty)=e^{*}$. By C.5, the integral (2.10) converges and we estimate the integral in (2.21),

$$
\begin{aligned}
\left\|H_{1}(t)\right\| & =\left\|\int_{t}^{\infty} G_{1}(\tau) d \tau\right\|=\left\|\int_{t}^{\infty} d \tau \int_{\tau}^{\infty} F_{1}(x(\sigma)) d \sigma\right\|=\left\|\iint_{t<\tau<\sigma} F_{1}(x(\sigma)) d \tau d \sigma\right\| \\
& \leqq \iint_{t<\tau<\sigma}\left\|F_{1}(x(\sigma))\right\| d \tau d \sigma=\int_{t}^{\infty}(\sigma-t)\left\|F_{1}(x(\sigma))\right\| d \sigma .
\end{aligned}
$$

By (2.33) and (2.34), $\left\|H_{1}(t)\right\|<\infty$ and $\left\|H_{1}(t)\right\| \rightarrow 0$ as $t \rightarrow \infty$. The rest of the proof of Theorem 1 goes through unchanged and the obtained contradiction proves Proposition 1.

The following lemma is used in Sect. 3.

Lemma 1. Let $E \sim R^{N}$ be a Euclidean space with the scalar product $\langle x, y\rangle$ and let 
$E_{i} \sim R^{N_{i}}, i=1, \ldots, n$, be a nonempty family of subspaces of $E$. Denote by $P_{i}$ the orthogonal projection of $E$ onto $E_{i}$.

Let for $i=1, \ldots, n$ a potential $V_{i}$ in $\Omega_{i} \subset E_{i}$ be given. Denote by $C_{i} \subset E_{i}$ the cone spanned by vectors $F_{i}(x)=-\operatorname{grad} V_{i}$ and assume that for $i=1, \ldots, n$, conditions C.1-C.3 are satisfied. Set $\Omega=\left\{x \in E: P_{i}(x) \in \Omega_{i}, i=1, \ldots, n\right\}$ and

$$
V(x)=\sum_{i=1}^{n} V_{i}\left(P_{i} x\right)
$$

If the cone $C$ in $E$ spanned by $C_{1}, \ldots, C_{n}$ is proper, then $V$ satisfies conditions $C .1-C .3$.

Proof. The dual cone $C^{*}$ is given by

$$
C^{*}=\left\{x \in E: P_{i} x \in C_{i}^{*}, \quad i=1, \ldots, n\right\} .
$$

If $x \in \Omega$ and $e^{*} \in C^{*}$, then for $i=1, \ldots, n, P_{i} x \in \Omega_{i}$ and $P_{i}\left(x+e^{*}\right) \in \Omega_{i}+C_{i}^{*} \subset \Omega_{i}$. This shows that $\Omega$ contains $\Omega+C^{*}$. If $e \in C$ then $e=e_{1}+\cdots+e_{n}$, where $e_{i} \in C_{i}$ and

$$
\langle x, e\rangle=\sum_{i=1}^{n}\left\langle x, e_{i}\right\rangle=\sum_{i=1}^{n}\left\langle P_{i} x, e_{i}\right\rangle \text {. }
$$

Assume, for simplicity of exposition, that $e_{i} \neq 0$ for $1 \leqq i \leqq n$, and let $l_{i} \geqq-\infty$ be the lower bound on $\left\langle P_{i} x, e_{i}\right\rangle$. Set $l=\sum_{i=1}^{n} l_{i}$. Then $\left.\langle x, e\rangle\right\rangle l$.

Assume first that $l>\infty$, i.e. $l_{i}$ is finite for all $i$. Then $\langle x, e\rangle \rightarrow l$ if and only if $\left\langle P_{i} x, e_{i}\right\rangle \rightarrow l_{i}$ for all $i$. The latter implies that $V_{i}\left(P_{i} x\right) \rightarrow \infty$ for all $i$, hence $V(x) \rightarrow \infty$. The equality $l=-\infty$ holds if and only if $l_{i}=-\infty$ for at least one index $i$. We have $\langle x, e\rangle \rightarrow-\infty$ if and only if $\left\langle P_{i} x, e_{i}\right\rangle \rightarrow-\infty$ for at one of those $i$, say for $i=1$. But then $V_{1}\left(P_{1} x\right) \rightarrow \infty$ implying $V(x) \rightarrow \infty$.

Analogously, if $\langle x, e\rangle$ is bounded from above, then for at least one index $i,\left\langle P_{i} x, e_{i}\right\rangle$ is bounded from above, therefore $\left\|F_{i}(x)\right\|=\left\|F_{i}\left(P_{i} x\right)\right\|$ is bounded away from zero. Since $C_{1}, \ldots, C_{n}$ span a proper cone, there is a constant $a>0$ such that for any $e_{i} \in C_{i}, i=1, \ldots, n$, we have

$$
\left\|e_{1}+\cdots+e_{n}\right\| \geqq a\left(\left\|e_{1}\right\|+\cdots+\left\|e_{n}\right\|\right) .
$$

By these two observations, $\|F(x)\|$ is bounded away from zero. So far we have proved C.1 and C.2. It remains to prove that C.3 is satisfied.

A vector $e^{*} \in C^{*}$ defines $P_{i} e^{*}=e_{i}^{*} \in C_{i}^{*}$ and let $C_{i}^{0}$ and $C_{i}^{1}$ be the corresponding subcones of $C_{i}$. Since for any vector $e=\sum_{i=1}^{n} e_{i}$ in $C$,

$$
\left\langle e, e^{*}\right\rangle=\sum_{i=1}^{n}\left\langle e_{i}, e_{i}^{*}\right\rangle=0
$$

if and only if $\left\langle e_{i}, e_{i}^{*}\right\rangle=0$ for all $i$, the cone $C_{0}$ is the span of $C_{i}^{0}, i=1, \ldots, n$. Set $C_{1}$ be the span of $C_{i}^{1}$ for $i=1, \ldots, n$, and define the decomposition (2.3) by

$$
V(x)=\sum_{i=1}^{n} V_{i}\left(P_{i} x\right)=\sum_{i=1}^{n} V_{i}^{0}\left(P_{i} x\right)+\sum_{i=1}^{n} V_{i}^{1}\left(P_{i} x\right)=V_{0}(x)+V_{1}(x) .
$$


In a selfexplanatory notation, we have

$$
F_{0}(x)=\sum_{i=1}^{n} F_{i}^{0}\left(P_{i} x\right) \in C_{0}, \quad F_{1}(x)=\sum_{i=1}^{n} F_{i}^{1}\left(P_{i} x\right) \in C_{1} .
$$

Since $e_{i}^{*}=P_{i} e^{*}$ belongs to $\operatorname{Int} C_{i}^{1}, e^{*} \in \operatorname{Int} C_{1}$.

It remains to show convergence of the integral (2.4). Using notation $a_{i}=P_{i} a$, we have

$$
\int_{0}^{\infty} V_{1}\left(a+r e^{*}\right) d r=\sum_{i=1}^{n} \int_{0}^{\infty} V_{i}^{1}\left(a_{i}+r e_{i}^{*}\right) d r<\infty
$$

which finishes the proof.

\section{Applications and Examples}

Applications of the results of Sect. 2 to the many-body scattering are based on the following theorem which is a straightforward corollary of Theorem 1 and Lemma 1.

Theorem 2. Let $E$ be a Euclidean space of $N$ dimensions with orthogonal projections $P_{1}, \ldots, P_{n}$ on the subspaces $E_{1}, \ldots, E_{n}$. Let

$$
V(x)=\sum_{i=1}^{n} V_{i}\left(P_{i} x\right)
$$

be a potential such that for $i=1, \ldots, n$ the potentials $V_{i}$ on $E_{i}$ satisfy C.1.-C.3 with domains $\Omega_{i}$ and cones $C_{i}$ in $E_{i}$. Denote by $C$ the cone in $E$ spanned by $C_{1}, \ldots, C_{n}$.

If $C$ is proper then all trajectories $x(t)$ of Newton's equation

$$
M \ddot{x}=-\operatorname{grad} V(x)
$$

are regular with $\dot{x}(\infty) \in \operatorname{Int} C^{*}, \dot{x}(-\infty) \in-\operatorname{Int} C^{*}$.

Theorem 3. Let $e_{1}, \ldots, e_{n}$ be nonzero vectors in $E \sim R^{N}$ spanning a proper cone $C$. Let $v_{i}(r)$ be continuously differentiable functions defined in $\left\{-\infty \leqq a_{i}<r<\infty\right\}$ for $i=1, \ldots, n$. Assume that $v_{i}(r)$ monotonically decreases from infinity to zero when $r$ runs from $a_{i}$ to $\infty$. Assume also that $f_{i}(r)=-v_{i}^{\prime}(r)>0$ and that for $A_{i}>a_{i}$,

Set

$$
\int_{A_{i}}^{\infty} v_{i}(r) d r<\infty
$$

$$
V(x)=\sum_{i=1}^{n} v_{i}\left(\left\langle x, e_{i}\right\rangle\right)
$$

Then the trajectories of the dynamics (3.1) are regular and satisfy

$$
\left\langle\dot{x}(\infty), e_{i}\right\rangle>0,\left\langle\dot{x}(-\infty), e_{i}\right\rangle<0
$$

for $i=1, \ldots, n$.

Proof. The situation of Theorem 3 is a special case of that of Theorem 2 with $E_{i}=R e_{i}, C_{i}=R_{+} e_{i}, \Omega_{i}=\left(a_{i}, \infty\right)$. It is trivial to check that for $i=1, \ldots, n$, potentials $v_{i}$ satisfy C.1-C.3. Hence, Theorem 2 applies. 
Example 1. Exponential Cone Potentials. These are the potentials of the form [3]

$$
V(x)=\sum_{i=1}^{n} c_{i} e^{-\left\langle x, e_{i}\right\rangle},
$$

where $c_{i}>0$ and the vectors $e_{1}, \ldots, e_{n}$ in $E$ span a proper cone. The following is a special case of Theorem 3 .

Corollary 3. Any trajectory $x(t)$ of the dynamics (3.1) with the exponential potential $V$ given by (3.5) is regular. The velocities $\dot{x}(\infty), \dot{x}(-\infty)$ satisfy (3.4).

Potential $V$ given by (3.3) is a linear superposition of potentials $v_{i}$ in one dimension. Now we consider the polynomial superpositions of such potentials. Let $e_{i} \neq 0$ be a vector in $E \sim R^{N}, i=1, \ldots, N$. By polynomial superposition of $v_{i}$ we mean potentials $V$ on $E$ of the form

$$
V(x)=\sum_{m} \sum_{I=\left(i_{1}, \ldots, i_{m}\right)} c_{I} v_{i_{1}}\left(\left\langle x, e_{i_{1}}\right\rangle\right) \cdots v_{i_{m}}\left(\left\langle x, e_{i_{m}}\right\rangle\right)
$$

where the summation is over a finite set of multi-indices $I$ and $c_{I}>0$.

Theorem 4. Let a potential $V$ on $E$ be a polynomial superposition (3.6) of potentials $v_{i}$ in one dimension satisfying conditions of Theorem 3. If the cone $C$ in $E$ spanned by $e_{1}, \ldots, e_{N}$ is proper then the motions $x(t)$ of the dynamics (3.1) are regular with $\dot{x}(\infty) \in \operatorname{Int} C^{*}, \dot{x}(-\infty) \in-\operatorname{Int} C^{*}$.

Proof. The cone $C^{*}$ is given by $C^{*}=\left\{x:\left\langle x, e_{i}\right\rangle \geqq 0, i=1, \ldots, N\right\}$, the domain $\Omega$ by

$$
\Omega=\left\{x:\left\langle x, e_{i}\right\rangle>a_{i}, \quad i=1, \ldots, N\right\} .
$$

The force $F(x)=-\operatorname{grad} V(x)$ has expansion

$$
F(x)=\sum_{I} \sum_{i \in I} c_{I} v_{i_{1}}\left(\left\langle x, e_{i_{1}}\right\rangle\right) \cdots v_{i_{m}}\left(\left\langle x, e_{i_{m}}\right\rangle\right) f_{i}\left(\left\langle x, e_{i}\right\rangle\right) e_{i} .
$$

Conditions C.1 and C.2 are elementary to check. Condition C.4 is fairly straightforward and we leave it to the reader.

For $e^{*} \in C^{*}$ let $K_{0}$ be the set of indices $i$ such that $\left\langle e^{*}, e_{i}\right\rangle=0$, and let $K_{1}$ be the complement of $K_{0}$. Set $F_{0}(x)$ be the part of the expansion (3.7), where $i \in K_{0}$ and $F_{1}(x)$ be the rest of the expansion. Since $C_{0}$ is spanned by $e_{i}, i \in K_{0}$, we have $F_{0}(x) \in C_{0}$. To obtain C.5, it suffices to bound the integrals

$$
\int_{T}^{\infty} t v_{i_{1}}\left(\left\langle x(t), e_{i_{1}}\right\rangle\right) \cdots v_{i_{m}}\left(\left\langle x(t), e_{i_{m}}\right\rangle\right) f_{i}\left(\left\langle x(t), e_{i}\right\rangle\right) d t
$$

for any multi-index $I=\left(i_{1}, \ldots, i_{m}\right), i \in I \cap K_{1}$ and $T$ sufficiently large. By conservation of energy, $v_{j}\left(\left\langle x(t), e_{j}\right\rangle\right)$ is bounded on the trajectory $x(t)$ for any $j$, therefore $\left\langle x(t), e_{j}\right\rangle$ is bounded away from $a_{j}$, hence in the integral (3.8) the product $v_{i_{1}}\left(\left\langle x(t), e_{i_{1}}\right\rangle\right) \cdots$ $v_{i_{m}}\left(\left\langle x(t), e_{i_{m}}\right\rangle\right)$ is bounded as $t \rightarrow \infty$. Since $i \in K_{1}, \lim _{t \rightarrow \infty}\left\langle\dot{x}(t), e_{i}\right\rangle>0$ and we can find $a>0$ such that $\left\langle x(t), e_{i}\right\rangle \geqq a t$ for $t$ large enough. Hence, (3.8) is bounded above by

$$
\text { const } \int_{T}^{\infty} t f_{i}(a t) d t
$$


Integrating (3.9) by parts and using that, in view of (3.2)

$$
\lim _{r \rightarrow \infty} r v_{i}(r)=0 \text {, }
$$

we conclude that (3.9) converges. Thus, Proposition 1 applies.

Example 2. Polynomial Superpositions of $x^{-2}$ and $e^{-x}$ Potentials. Let vectors $e_{i} \neq 0$, $i=1, \ldots, N$, span a proper cone $C$ in $E$. Let $a_{i}, b_{j}, c_{i j}, 1 \leqq i, j \leqq N$ be nonnegative numbers, not all of them zero. Consider the potential

$$
V(x)=\sum_{i=1}^{N} a_{i} e^{-\left\langle x, e_{i}\right\rangle}+\sum_{j=1}^{N} b_{j}\left\langle x, e_{j}\right\rangle^{-2}+\sum_{i, j=1}^{N} c_{i j}\left\langle x, e_{i}\right\rangle^{-2} e^{-\left\langle x, e_{j}\right\rangle},
$$

defined in the domain Int $C^{*}$ and the corresponding dynamics (3.1). Theorem 4 implies the following.

Corollary 4. Trajectories $x(t)$ of the dynamics (3.1) with potential (3.11) are all regular and $\dot{x}(\infty) \in \operatorname{Int} C^{*}, \dot{x}(-\infty) \in \operatorname{Int} C^{*}$.

Remark. One can consider, analogously, more general than (3.11) superpositions of $x^{-2}$ and $e^{-x}$ potentials.

In the rest of this section we consider applications to the many-body problems on the line. We denote by $x_{1}, \ldots, x_{N}$ the positions, by $\dot{x}_{1}, \ldots, \dot{x}_{N}$ the velocities and by $m_{1}, \ldots, m_{N}$ the masses of the particles. We assume that the particles interact pairwise by conservative repulsive forces and consider the following two cases.

Case 1. Central Forces. The pair potentials depend on the distances between particles, $v_{i j}\left(\left|x_{i}-x_{j}\right|\right)$. We assume that the potentials $v_{i j} \geqq 0,1 \leqq i<j \leqq N$, are continuously differentiable on $(0, \infty)$, monotonically decreasing and that $\lim v_{i j}(r)=$ 0 as $r \rightarrow \infty$. The total potential in the case of central forces is

$$
V\left(x_{1}, \ldots, x_{N}\right)=\sum_{i<j} v_{i j}\left(\left|x_{i}-x_{j}\right|\right)
$$

Case 2. Directed Forces. The pair potentials are $v_{i j}\left(x_{i}-x_{j}\right), 1 \leqq i<j \leqq N$, where $v_{i j}(r) \geqq 0$ is continuously differentiable on the interval $\left\{-\infty \leqq l_{i j}<r<\infty\right\}$ and monotonically decreases from infinity to zero.

Theorem 5. Let $N$ particles on the line interact by central repulsive forces. Assume that all pair potentials $v_{i j}(r) \rightarrow \infty$ when $r \rightarrow 0$, that $v_{i j}^{\prime}<0$ and that for $\rho>0$ the integrals $\int_{\rho}^{\infty} v_{i j}(r) d r$ converge.

Then every trajectory $\left\{x_{1}(t), \ldots, x_{N}(t)\right\}$ is regular and $\dot{x}_{1}(\infty) \neq \cdots \neq \dot{x}_{N}(\infty)$.

Proof. The condition $v_{i j}(0)=\infty$ means that the particles cannot pass each other. Therefore on a given trajectory we can relabel them in such a way that for all times

$$
x_{1}>\cdots>x_{N} .
$$

Let $e_{1}, \ldots, e_{N}$ be the standard basis of $R^{N}$ and let $C$ be the cone spanned by $e_{1}-e_{2}, \ldots, e_{N-1}-e_{N}$. Then the total potential (3.12) in the domain (3.13), which 
is Int $C^{*}$, takes the form (3.3)

$$
V(x)=\sum_{i<j} v_{i j}\left(\left\langle x, e_{i}-e_{j}\right\rangle\right)
$$

and satisfies conditions of Theorem 3, which implies the assertion.

Remarks. 1. What happens if the particles can pass each other, i.e. $v_{i j}(0)<\infty$ for some $i<j$ ? By [1] and [9], the asymptotic velocities $\dot{x}_{1}(\infty), \ldots, \dot{x}_{N}(\infty)$ exist for every motion. By [2], the motions satisfying (1.4) are asymptotically uniform (1.5), but now the regularity condition (1.4) is not satisfied by all trajectories, only by generic ones. For instance, if $v_{i j}(0)<\infty$ for all $i<j$ then there are relative equilibria

$$
x_{1}(t)=\cdots=x_{N}(t)=a+b t, \quad \dot{x}_{1}(\infty)=\cdots=\dot{x}_{N}(\infty)=b
$$

which violate (1.4). Nevertheless, the motions (3.14) are asymptotically uniform. Are there trajectories which are not asymptotically uniform? The question seems to be open, see [2].

2. Theorem 5 obviously extends to the case when the pair potentials $v_{i j}$ are defined on $\left(a_{i j}, \infty\right)$ with $a_{i j} \geqq 0$, and the other conditions remain the same.

Now we consider $N$-body problems with directed forces and allow, in addition, external potentials $u_{i} \geqq 0$ which are continuously differentiable for $-\infty \leqq l_{i}<r<$ $\infty$ and monotonically decrease from infinity to zero. The following is a corollary of Theorem 3.

Theorem 6. Let

$$
V\left(x_{1}, \ldots, x_{N}\right)=\sum_{i<j} v_{i j}\left(x_{i}-x_{j}\right)+\sum_{k=1}^{N} u_{k}\left(x_{k}\right)
$$

be the total potential of a many-body problem with directed forces. Assume that $v_{i, i+1}^{\prime}<0$ for $i=1, \ldots, N-1$ and that for all $i<j$ and $\rho>l_{i j}$ the integrals $\int_{0}^{\infty} v_{i j}(r) d r<\infty$. Also assume that for $k=1, \ldots, N$, either $u_{k}=0$ or $u_{k}^{\prime}<0$ and $\rho$ $\int_{\rho}^{\infty} u_{k}(r) d r<\infty$

Then every trajectory of the $N$-body problem is regular and $\dot{x}_{1}(\infty)>\cdots>\dot{x}_{N}(\infty)$. Let $k_{0}$ be the minimal $k$ such that $u_{k} \neq 0$. Then for $k \geqq k_{0}, \dot{x}_{k}(\infty)>0$.

Remark. One of the results of [7] is a criterion of regularity of asymptotic velocities for a class of repulsive many-body interactions in one dimension. Assume that the particles have equal masses (and set $m=1$ ), and that the directed repulsive pair potentials $v_{i j}$ are all equal. Then the Hamiltonian of the system is

$$
H=\frac{1}{2} \sum_{k=1}^{N} \dot{x}_{k}^{2}+\sum_{i<j} v\left(x_{i}-x_{j}\right) .
$$

Assume that the interaction potential $v$ is continuously differentiable on $(l, \infty)$.

Theorem [7]. The usual repulsivity conditions on $v$ (i.e. $v>0, v^{\prime}<0$ and $\lim v(x)=\infty$ 
as $x \rightarrow l$ ) are necessary and sufficient for the property

$$
\dot{x}_{1}(\infty)>\cdots>\dot{x}_{N}(\infty)
$$

for all trajectories of the Hamiltonian (3.16).

Thus, for system (3.16), no decay conditions on $v$ are needed for the regularity (3.17) of asymptotic velocities.

\section{References}

1. Galperin, G.: On systems of locally interacting and repelling particles moving in space. Trans. Mos. Math. Soc. 43, 159-214 (1981)

2. Galperin, G.: Asymptotic behavior of particle motion under repulsive forces. Commun. Math. Phys. 84, 547-556 (1982)

3. Gutkin, E.: Integrable Hamiltonians with exponential potential. Physica 16D, 398-404 (1985)

4. Gutkin, E.: Asymptotic of trajectories for cone potentials. Physica 17D, 235-242 (1985)

5. Gutkin, E.: Continuity of scattering data for particles on the line with directed repulsive interactions. J. Math. Phys. 28, 351-359 (1987)

6. Gutkin, E.: Asymptotics of trajectories for Newtonian dynamics with directed force. Physica 26D 156164 (1987)

7. Hubacher, A.: Classical scattering theory in one dimension, preprint

8. Moser, J.: Three integrable Hamiltonian systems connected with isospectral deformations. Adv. Math. 16, 197-220 (1975)

9. Vaserstein, L. M.: On systems of particles with finite range and/or repulsive interactions. Commun. Math. Phys. 69, 31-56 (1979)

Communicated by J. Mather

Received January 26, 1987; in revised form December 11, 1987 